\title{
Assessment protocols for forward masking in Frequency-Following Response
}

\author{
Denise Costa Menezes ${ }^{1}$ \\ https://orcid.org/0000-0002-2008-4091 \\ Silvana Maria Sobral Griz ${ }^{1}$ \\ https://orcid.org/0000-0002-6759-5964 \\ Anne Karoline Lima de Araújo ${ }^{1}$ \\ https://orcid.org/0000-0003-0341-579X \\ Leonardo Gleygson Angelo Venâncio' \\ https://orcid.org/0000-0002-1971-755X \\ Karina Paes Advincula ${ }^{1}$ \\ https://orcid.org/0000-0002-1314-6100 \\ Pedro de Lemos Menezes ${ }^{2}$ \\ https://orcid.org/0000-0003-1999-5055
}

Universidade Federal de Pernambuco UFPE, Recife, Pernambuco, Brasil.

${ }^{2}$ Universidade Estadual de Ciências da Saúde de Alagoas - UNCISAL, Alagoas, Maceió, Brasil.

This project was carried out in the Postgraduate Program in Human Communication Disorders at the Universidade Federal de Pernambuco UFPE, Recife, Pernambuco, Brazil.

Research support source: Coordenação de Aperfeiçoamento de Pessoal de Nível Superior - Brasil - CAPES, Finance Code 001.

Conflict of interests: Nonexistent

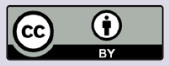

Received on: July 15,2019

Accepted on: August 25, 2020

Corresponding address:

Denise Costa Menezes

Avenida Professor Artur de Sá, 267 -

Cidade Universitária

CEP: 50740-020 - Recife, Pernambuco, Brasil

E-mail: denicmenezes@gmail.com

\section{ABSTRACT}

Purpose: to investigate forward masking by comparing latencies values of positive and negative peaks in frequency-following responses (FFR) recordings, in normally hearing young adults.

Methods: from a database, 20 FFR recordings were selected, 10 being from men, and 10 from women, aged 18 to 25 years, with normal hearing. They were qualitatively analyzed by two experienced researchers and also analyzed, according to two different protocols of recording identification: (i) predominance of positive peaks - PV, A, PW, $P X, P Y, P Z$, and 0 waves; and (ii) predominance of negative peaks $-V, A, C, D, E, F$, and 0 waves. The Shapiro-Wilk normality test, the Wilcoxon test, and the Student's t-test were conducted, by adopting the significance level of $p<0.05$.

Results: the comparative analysis of latency peak values did not reveal any significant difference between the studied protocols. However, the standard deviation was higher for absolute latency values as compared to negative peaks, suggesting an inverted pattern of what was expected.

Conclusion: forward masking was identified in both proposals and the protocol of predominant positive peaks was less variable.

Keywords: Speech Perception; Perceptual Masking; Noise; Auditory-Evoked Potentials; Protocols; Electrophysiology 


\section{INTRODUCTION}

In everyday situations, hearing can be a challenging task even for people with normal hearing thresholds. In most environments, speech occurs simultaneous to various types of noise - energy with the potential to degrade the temporal structure of the target sound. When the target sound is speech, the noise frequently makes it unintelligible. Consequently, the complaint of not understanding speech in noisy environments, even for normally hearing people, is related to the temporal auditory processing, which may be impaired'.

Frequency-following responses (FFR) can be elicited by a complex stimulus, such as a speech sound. The most commonly used speech stimulus in FFR recordings is the /da/ syllable. It allows the speech sound to be decoded throughout the whole auditory system².

FFR records are usually analyzed in time domain, whose peaks are marked by the examiner, normally identifying positive and/or negative peaks. Two forms of identifying peaks have been previously described: (i) analysis with predominantly identified positive peaks, according to Hodge et al. (2018) ${ }^{3}$, in which the PV, A, PW, PX, PY, PZ, and $\mathrm{O}$ waves are identified; and (ii) analysis with predominantly identified negative peaks, according to Skoe and Kraus (2010) ${ }^{2}$, in which the V, A, $C, D, E, F$, and $O$ waves are identified. In both, latency values are identical for the $V(P V), A$, and $O$ waves when the /da/ syllable is used.

In auditory evoked potentials recordings, latency values are commonly analyzed in the temporal domain. Absence or delays in wave latencies can indicate changes or impairment in processing complex sound stimuli on the auditory neural pathway ${ }^{3}$.

Hodge et al. ${ }^{3}$ observed more prominent positive peaks than the negative peaks. Instead of selecting negative peak responses, this study used positive peak label to measure latencies, especially to investigate forward masking. Investigation of two FFR identifying protocols proposed in this study: (i) analysis of PV, A, PW, PX, PY, PZ, and O peak identification; and (ii) analysis of $P V, A, C, D, E, F$, and $O$ peak identification.

The focus is a better understanding of how the auditory system reacts to difficult listen situations, such as in presence of noise. The interest in investigating the decoding of complex verbal sounds, such as speech. Changed response patterns have been observed in people with language problems ${ }^{4}$, allowing for differential diagnosis and therapeutic monitoring. For instance, abnormal FFR latencies can be found in individuals with hearing loss $^{5}$, children with learning difficulties, and auditory processing changes ${ }^{6,7}$. Therefore, FFR is considered an objective method to assess the auditory processing mechanisms, used as a biological marker of decoding speech sounds ${ }^{3}$.

FFR can be used in the studies of temporal masking a phenomenon related to temporal auditory processing and, consequently, to understand speech in noisy environments ${ }^{3,8}$. Temporal masking is characterized by increasing in sound threshold due to presence a more intense sound. It occurs when a stimulus (masking noise) is presented with duration and intensity enough to reduce the sensitivity of the target sound. The noise can be presented simultaneously, before, or after the target stimulus. Thus, the audibility of a target sound is modified by the presence of a simultaneous or nonsimultaneous masking noise. The later either backward or forward masking ${ }^{9}$.

In forward masking, the noise is presented before the target sound (such as, speech stimulus), producing a masking effect that extends for some milliseconds after its acoustic presentation has finished ${ }^{10,11}$. This noise's permanence effect disturbs the processing of the subsequent speech signal ${ }^{11}$. Forward masking studies is related to speech perception in noisy environments. However, it is not clear which is the best analysis method for FFR.

This study investigated how forward masking occurs with positive and with negative peak identification, comparing FFR latency values in young adults with normal hearing.

\section{METHODS}

This research was developed in the Audiology Laboratory of the Department of Speech-LanguageHearing Sciences at the Universidade Federal de Pernambuco - UFPE, PE, Brazil. It was approved by the Human Research Ethics Committee under protocol number 1.727.677. It is an exploratory descriptive study.

From a database, 20 FFR recordings were selected - 10 from male and 10 from female young adults, aged 18 to 25 years, with pure tone audiometry thresholds equal to or better than $25 \mathrm{~dB} \mathrm{HL}$ at the frequencies between 500 and $8000 \mathrm{~Hz}$, with no history of audiological complaints of peripheral or central processing difficulties, neurological difficulties, and otologic changes. The auditory processing changes were excluded based on the absence of a diagnosis or report of audiological changes. 
Data were selected from Intelligent Hearing Systems database through a qualitative analysis of the recordings by two examiners with expertise in FFR assessment. For the recordings, participants were accommodated on a reclining chair in a sound booth. The skin was prepared with abrasive paste and the electrodes were fixed according to the 10-20 International System: positive electrode at Fz (upper forehead); negative electrodes at M1 (left mastoid) and M2 (right mastoid), and the ground electrode at Fpz (lower forehead). The stimuli were presented monaurally at the right ear via E39 insert earphones. Two protocols were used: (1) no masking - /da/ syllable alone, with duration of $40 \mathrm{~ms}$, originated from the Northwestern University laboratory, with alternating polarity, presented at 3.77/second, at $75 \mathrm{~dB}$ SPL; (2) with masking - the same /da/ syllable (with duration of $40 \mathrm{~ms}$, originated from the Northwestern University laboratory, with alternating polarity, presented at 3.77/ second, at $75 \mathrm{~dB} \mathrm{SPL}$ ) presented $4 \mathrm{~ms}$ after a shaped speech noise (SSN) noise (with $100 \mathrm{~ms}-10 \mathrm{~ms}$ rise/ fall time, at $80 \mathrm{~dB} S P L$ - originated from the Auditory Sciences Laboratory at the University of North Carolina at Chapel Hill) ${ }^{3}$. The recording window was $70 \mathrm{~ms}$, with low-pass and high-pass filters, respectively, at $3000 \mathrm{~Hz}$ and $50 \mathrm{~Hz}$. Three or more traces, with 2000 sweeps, were conducted, and the analysis was made at the resulting trace (6000 stimuli - the sum of the three best tracings).

The tracings were analyzed following the protocols by (i) Hodge et al. (2018): PV, A, PW, PX, PY, PZ, and O waves; and (ii) Skoe and Kraus (2010): V, A, C, D, E, $F$, and $O$ waves. The latency values of $P V(V), A$, and $O$ waves were identical in both protocols, as they did not change between them.

Latencies mean values and standard deviation were determined for both FFR assessment protocols. The Shapiro-Wilk normality test was preformed showing normal and not normal data distribution. In order to compare positive and negative peaks with normal distibrution, Wilcoxon test was perfomed, and to compare positive and negative peaks with no normal distribution, a t- Student was performed. To evaluate correlation among variable values, the Spearman's correlation test was performed to non normal data, and the Pearson's correlation test was performed to evaluate normal data. For all tests, $5 \%$ level of significance was adopted.

\section{RESULTS}

In order to investigate forward masking, both wave picking protocols (positive peaks - PW, PX, PY, and PZ; and negative peaks - C, D, E, and F), in both testing conditions (no masking and noise presented 4 millisecond before the /da/ syllable) were used (Figure 1).

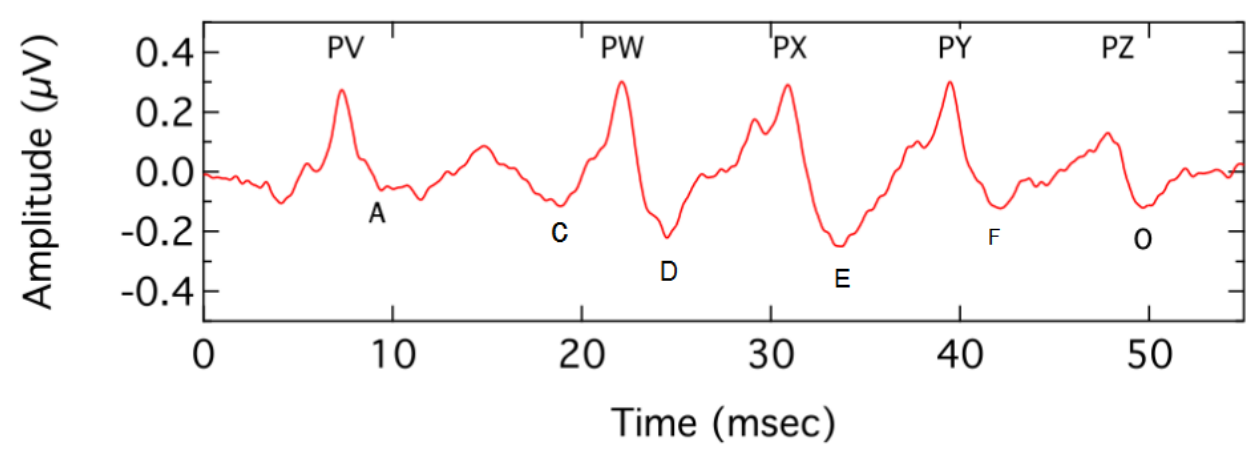

Figure 1. Different forms of frequency-following response peak identifying: predominance of positive and negative peaks

Table 1 shows FFR latency values obtained by identifying positive and negative peaks, as well as the latency difference values obtained in the two conditions (with and without masking. Positive peaks did not show a normal distribution, and pairs were compared using the Wilcoxon test. Negative peaks shoed normal distribution and pairs were compared using t-student test. The forward masking magnitude was obtained by the difference between the mean latency values of each conditions. 
Table 1. Latency values and standard deviation of the frequency-following response waves obtained in positive and negative peaks, and latency difference values obtained in both /da/ syllable presentation conditions (with and without masking)

\begin{tabular}{|c|c|c|c|c|c|c|}
\hline Condition & Wave & Mean (ms) & SD & Wave & Mean (ms) & SD \\
\hline \multirow{4}{*}{ WoM } & PW & 22.22 & 0.47 & C & 18.06 & 1.10 \\
\hline & PX & 30.71 & 0.71 & D & 24.37 & 0.89 \\
\hline & PY & 39.34 & 0.54 & $E$ & 33.38 & 1.20 \\
\hline & $\mathrm{PZ}$ & 47.91 & 0.63 & $\mathrm{~F}$ & 41.77 & 1.24 \\
\hline \multirow{4}{*}{ WM } & PW & 22.79 & 0.52 & $C$ & 18.70 & 1.52 \\
\hline & PX & 31.54 & 0.40 & D & 24.72 & 1.36 \\
\hline & PY & 40.15 & 0.75 & $E$ & 33.89 & 1.38 \\
\hline & PZ & 48.58 & 0.69 & $\mathrm{~F}$ & 42.49 & 1.61 \\
\hline \multirow{4}{*}{ Dif } & $\operatorname{dif} P W^{*}$ & 0.57 & 0.30 & $\operatorname{dif} C^{* \star}$ & 0.64 & 1.71 \\
\hline & $\operatorname{dif} P X^{*}$ & 0.83 & 0.60 & $\operatorname{dif} D^{* *}$ & 0.35 & 0.83 \\
\hline & $\operatorname{dif} P Y^{*}$ & 0.81 & 0.99 & $\operatorname{dif} E^{\star *}$ & 0.52 & 0.80 \\
\hline & $\operatorname{dif} \mathrm{PZ}^{\star}$ & 0.67 & 0.52 & $\operatorname{dif} F^{* *}$ & 0.72 & 1.29 \\
\hline
\end{tabular}

* Wilcoxon (Significance level adopted: $p<0,05$ )

** T-student test for pairs (Significance level adopted: $p<0,05$ )

Legend:

WoM: Presentation of the /da/ syllable without masking

WM: Presentation of the /da/ syllable 4 milliseconds after presenting the masking noise

SD: Standard deviation

Dif*: Forward masking magnitude (the difference between both stimulus presentation conditions)

The positive peaks PX, PY, and PZ waves did not present a normal distribution, tested by Shapiro-Wilk test. Therefore, parametric tests could not be used to compare the results obtained with the negative peaks (D, E, and $\mathrm{F}$ waves) protocol, which had a normal distribution. Thus, the Wilcoxon test was performed. The significance level of $p<0.05$ was used. However, the PW result presented a normal distribution; hence, it was compared with the subsequent negative $C$ peak by t-student test (Table 2). No significant difference was observed in forward masking between the protocol.

Table 2 also shows the forward masking standard deviation (variations) for both protocols. Greater variability was observed for negative peaks analysis, especially for wave $C(S D=1.44)$ and $F(S D=1.29)$.

Spearman's correlation test was used for PX/D, $\mathrm{PY} / \mathrm{E}$, and $\mathrm{PZ} / \mathrm{F}$, as well as Pearson's vorrelation test, for the PW/C waves.

Table 2. Comparison of the differences between the latencies in the /da/ syllable presentation conditions (with and without masking) between the peak identifying protocol with a predominance of the positive peaks and negative peaks

\begin{tabular}{cccccc}
\hline Comparison & $\mathbf{n}$ & Mean $(\mathbf{m s})$ & Standard Deviation & Standard Error & Significance \\
\hline PW & 14 & 0.57 & 0.30 & 0.08 & $0.90^{\mathrm{b}}$ \\
C & 14 & 0.64 & 1.71 & 0.47 & \\
PX & 20 & 0.83 & 0.60 & 0.13 & $0.14^{\mathrm{a}}$ \\
D & 20 & 0.35 & 0.83 & 0.18 & $0.05^{\mathrm{a}}$ \\
PY & 20 & 0.81 & 0.99 & 0.22 & \\
E & 20 & 0.52 & 0.80 & 0.17 & $0.60^{\mathrm{a}}$ \\
PZ & 20 & 0.67 & 0.52 & 0.11 & 0.28 \\
F & 20 & 0.72 & 1.29 & & \\
\hline
\end{tabular}

a. Spearman's Correlation Test

b. Pearson's Correlation Test 
There were no significant correlations in any of the waves analyzed.

\section{DISCUSSION}

Forward masking investigation showed no difference between positive peaks negative peaks analysis. In other words, forward masking was the same according to peak analyze proposed by Hodge et al. ${ }^{3}-\mathrm{PV}, \mathrm{A}, \mathrm{PW}$, PX, PY, PZ, and $O$ waves - or according to what it was proposed by Skoe and $\mathrm{Kraus}^{2}-\mathrm{V}, \mathrm{A}, \mathrm{C}, \mathrm{D}, \mathrm{E}, \mathrm{F}$, and O waves.

However, analyzes of individual latency values, in the different testing conditions (with and without masking), some subjects presented greater latencies in the no-masking condition when using the negative peaks protocol (C, D, E, and F), resulting in inverting pattern. This called attention, considering the concept of forward masking, which is characterized by the wave latency delay in presence of noise. Yet, when analyzing positive peaks, forward masking was found in all situations.

This inverted response pattern (greater latencies on the no-masking condition when negative peaks were analyzed) may be the reason for values dispersion, resulting in greater standard deviation when negative peaks was analyzed. So, negative peaks identification presents less forward masking evidence, with less uniform values.

These results are consistent with Hodge et al. ${ }^{3}$, whose aim was to develop a forward masking analysis protocol. The authors have recorded FFR simultaneously in three different equipment (IHS, Biologic, and Neuroscan). Despite the same responses recorded in the three systems, identifying positive peaks was more consistent than identifying negative peaks, considering identical testing conditions.

The results found here suggest that using FFR positive peaks to analyze forward masking seems to lead to more visible waves, with and without masking. However, it does not reject the possibility of marking the negative peaks.

In the present study, the latency values differed from what had been previously described ${ }^{4-7}$, possibly due to the differences in acquisition protocols such as filters, stimuli presentation rate, intensity, etc. Nonetheless, the protocol used has been conceptualized as being ideal to obtain clearer responses (lower presentation rates $(3.77 / \mathrm{sec})$, greater intensities (75 dB SPL) of the stimulus and lower frequency spectrum of the filters $(100-1500 \mathrm{~Hz}))^{11}$, as a result, improves trace's morphology.

Since the identification of the FFR waves was based on the researchers' observation, biased resultos must be considered, despite the experience of the researchers.

Different FFR analyzes must be addressed in further studies, as the differences between the proposed protocols (predominance of positive and negative peaks) are yet not clear ${ }^{12-14}$.

\section{CONCLUSION}

Forward masking can be observed in both peak identifying protocols (either positive or negative peaks). However, FFR forward masking investigation using positive peaks protocol, in normally hearing young adults, suggests a smaller variability when compared to the negative peaks protocol.

\section{REFERENCES}

1. Pienkowski M. On the etiology of listening difficulties in noise despite clinically normal audiograms. Ear Hear. 2017;38(2):135-48.

2. Skoe E, Kraus N. Auditory brainstem response to complex sounds: a tutorial. Ear Hear. 2010;31(3):302-24.

3. Hodge SA, Menezes DC, Brown KD, Grose $\mathrm{JH}$. Forward masking of the speech-evoked auditory brainstem response. Otol Neurotol. 2018;39(2):150-7.

4. Meyer SO, Krizman J, White-Schwoch T, Kraus $N$. Children with autism spectrum disorder have unstable neural responses to sound. Exp Brain Res. 2018;236(3):733-43.

5. Anderson S, Kraus $\mathrm{N}$. The potential role of the CABR in assessment and management of hearing impairment. Int $\mathrm{J}$ Otolaryngol. 2013;2013(604729):1-10.

6. Ferreira L, Gubiani MB, Keske-Soares M, Skarzynski PH, Sanfins MD, Biaggio EPV. Analysis of the components of frequency-following response in phonological disorders. Int $\mathrm{J}$ Pediatr Otorhinolaryngol. 2019;122(2019):47-51.

7. Ananthakrishnan $S$, Luo $X$, Krishnan A. Human frequency following responses to vocoded speech. Ear Hear. 2017;38(5):e256-e267. 
8. Mehraei G, Gallardo AP, Shinn-Cunningham BG, Dau T. Auditory brainstem response latency in forward masking, a marker of sensory deficits in listeners with normal hearing thresholds. Hearing research. 2017;2017(346):34-44.

9. Peixe BP, Silva DD, Biaggio EPV, Bruno RS, Sanguebuche TR, Garcia MV. Applicability of evoked auditory brainstem responses with complex stimuli in adults with hearing loss. Int Arch Otorhinolaryngol. 2018;22(3):239-44.

10. Advíncula KP, Menezes DC, Pacífico FA, Costa MLG, Griz SMS. Age effects in temporal auditory processing: modulation masking release and forward masking effect. Audiol., Commun. Res. 2018;23(e1861):1-6.

11. Grose JH, Menezes DC, Porter HL, Griz S. Masking period patterns \& forward masking for speechshaped noise: age-related effects. Ear Hear. 2016;37(1):48-54.

12. Vanfeusden FJ, Chesnay MA, Seven DMS. Envelope frequency following responses are stronger for high-pass than low-pass filtered vowels. Int J Audiol. 2019;58(6):355-62.

13. Necciari T, Laback B, Savel S, Ystad S, Balazs P, Meunier $S$ et al. Auditory time-frequency masking for spectrally and temporally maximally-compact stimuli. PloS one. 2016;11(11):e0166937.

14. Gunaway TS, Ambikairajah E, Epps J. Perceptual speech enhancement exploiting temporal masking properties of human auditory system. Speech Communcation. 2010;52(5):381-93. 